\title{
NOREPINEPHRINE REGULATION OF CEREBRAL GLYCOGEN UTILIZATION DURING SEIZURES AND ISCHEMIA ${ }^{1}$
}

\author{
SAMI I. HARIK, ${ }^{2}$ RAUL BUSTO, AND ELENA MARTINEZ
}

Department of Neurology, University of Miami School of Medicine, Miami, Florida 33101

Received September 14, 1981; Revised December 7, 1981; Accepted December 9, 1981

\begin{abstract}
Norepinephrine (NE) depletion of the cerebral cortex after lesion of the ipsilateral locus ceruleus (LC) causes abnormalities of cerebral oxidative metabolism when the cortex is stimulated to increased energy demand (Harik, S. I., J. C. LaManna, A. I. Light, and M. Rosenthal (1979) Science 206: 69-71; LaManna, J. C., S. I. Harik, A. I. Light, and M. Rosenthal (1981) Brain Res. 204: 87101). These abnormalities were exhibited as decreased mitochondrial reducing equivalent flow. One possible cause of this would be the decreased availability of oxidative metabolic substrates in the NE-depleted cortex. We therefore investigated the effect of unilateral LC lesion and the resultant depletion of ipsilateral endogenous NE on glycogen and other energy metabolites in the cerebral cortex of rats under three conditions: (1) at "rest," (2) when energy demand is increased markedly by seizures, and (3) during total cerebral ischemia. We report no differences in cerebral metabolites between NE-depleted and control hemispheres at "rest." In seizures and ischemia, however, the increase in the level of adenosine $3^{\prime}: 5^{\prime}$-monophosphate (cyclic AMP) and the breakdown of glycogen were impaired considerably in the NE-depleted cortex. The data suggest that depletion of central NE impairs cerebral glycogenolysis in response to increased energy demands and ischemia. Such impairment may be mediated via a cyclic AMP-related mechanism.
\end{abstract}

Glycogen is the largest source of energy reserve in the brain. Although the role of norepinephrine (NE) in the regulation of liver and muscle glycogenolysis is well established, the effect of NE on cerebral glycogen metabolism remains ambiguous (Estler and Ammon, 1967; Passonneau et al., 1971; Hoffmann et al., 1973; Edwards et al., 1974; Gross and Ferrendelli, 1980). Based on results derived from in vivo optical monitoring of changes in the reduction/oxidation (redox) ratio of mitochondrial respiratory chain components in the cerebral cortex, we suspected the lack of adequate provision of metabolic substrates in the NE-depleted cortex when it is stimulated to heightened metabolic requirements (Harik et al., 1979; LaManna et al., 1981).

To test this hypothesis, we investigated the effect of cortical NE depletion on levels of high energy phosphate compounds, substrates, and products of glucose metabolism in the cerebral cortex of the rat. Studies were

\footnotetext{
${ }^{1}$ This research was supported by United States Public Health Service Grants NS 16617 and NS 05820. We thank Drs. J. C. LaManna and M. Rosenthal for helpful discussions and critical review of the manuscript and Mrs. Gail Troost and Ms. Joan-e Marklow for expert help in the preparation of the manuscript.

${ }^{2}$ To whom all correspondence should be addressed at his present address: Department of Neurology, Case Western Reserve University School of Medicine, Cleveland, OH 44106.
}

carried out in the "rest" state, under conditions of markedly increased metabolic demands induced by seizures, and during total cerebral ischemia. NE depletion of the cerebral cortex was produced by the local stereotaxic infusion of 6-hydroxydopamine (6-OHDA) into the locus ceruleus (LC). Since NE innervation of the cerebral cortex originates almost entirely from the ipsilateral LC (Ungerstedt, 1971; Kobayashi et al., 1975), unilateral LC lesion produces marked depletion of NE in the ipsilateral cortex while leaving the contralateral cortex with its normal NE content as an internal control (LaManna et al., 1981; Harik et al., 1981).

We find no differences in high energy phosphate compounds, glycogen, glucose, and products of glycolysis between the ipsilateral and contralateral hemispheres under "rest" conditions. However, when oxidative metabolism was challenged by increased activity during seizures or by interruption of the delivery of oxygen and glucose during ischemia, we measured significantly lower glycogen levels and higher adenosine $3^{\prime}: 5^{\prime}$-monophosphate (cyclic AMP) levels in the contralateral hemisphere in comparison to the ipsilateral, NE-depleted hemisphere. We interpret these results to mean that central NE plays an important regulatory role in activating glycogenolysis caused by seizures and ischemia. The impairment of glycogen breakdown and cyclic AMP ac- 
cumulation in the NE-depleted cortex may be related causally.

\section{Materials and Methods}

Locus ceruleus lesion. Male Wistar rats, about $150 \mathrm{gm}$, were used in all experiments. Rats were allowed a minimum of 5 days acclimation to our animal housing facilities before LC lesion. They were housed, 2 per cage, under diurnal light conditions with free access to laboratory chow and water. The $\mathrm{LC}$ was lesioned unilaterally by the local stereotaxic infusion of $2.5 \mu \mathrm{g}$ of 6 -OHDA base under chloral hydrate anesthesia and after pargyline pretreatment as described previously (LaManna et al., 1981; Harik et al., 1981). Approximately equal numbers of right and left LC lesions were made. The rats in the control group were treated in the same manner as the lesioned rats except that the scalp was not incised and no intracranial infusion was performed. We have demonstrated previously that infusion of vehicle solution into the LC does not cause significant reduction of NE levels in the ipsilateral cerebral cortex in comparison to the noninfused side (LaManna et al., 1981).

Control and lesioned rats were returned to their cages and allowed to recover for 2 weeks before they were used in further experiments. The rats with LC lesions showed no gross behavioral abnormalities, and their body weight was not different from controls.

"Rest" conditions and seizure experiment. Anesthesia was induced by diethyl ether. When the rats lost their righting reflexes, they were tracheostomized quickly, paralyzed with $d$-tubocurarine $(1 \mathrm{mg} / \mathrm{kg}$, i.p.), and mechanically ventilated with a mixture of $30 \%$ oxygen, $69 \%$ nitrous oxide, and $1 \%$ halothane to maintain surgical anesthesia. Femoral arterial and venous cannulae were inserted, and a plastic funnel was sutured over the calvaria for the subsequent freezing of the brain in situ (Ponten et al., 1973). After the surgical procedures were completed, the incisions were infiltrated with Xylocaine, and halothane was discontinued. In preliminary experiments, failure to use halothane during surgery resulted in arterial hypertension and hyperglycemia. A 30-min stabilization period then was allowed during which the respiratory rate and tidal volume were adjusted to maintain arterial blood gases and $\mathrm{pH}$ values within the normal range, and the blood pressure was stable. Body temperature was kept at $37^{\circ} \mathrm{C}$ by a rectal thermocouple and a heating pad.

Five rats with unilateral LC lesion were used for the determination of cerebral metabolites at "rest." In these rats, the brain was frozen in situ by pouring liquid nitrogen into the cranial funnel at the end of the stabilization period.

Seven rats with unilateral LC lesion and 4 control rats were used for the seizure experiment. Four brass electrodes applied to the skull provided bipolar EEG recordings from the two cerebral hemispheres. At the end of the stabilization period, nitrous oxide inhalation was stopped and ventilation was continued with $100 \%$ oxygen. One minute later, status epilepticus was induced by the intravenous injection of bicuculline, $1.2 \mathrm{mg} / \mathrm{kg}$ (Chapman et al., 1977). After 8 to $9 \mathrm{~min}$ of continuous electrical seizure activity, the brain was frozen in situ by pouring liquid nitrogen into the cranial funnel. Arterial blood pressure was monitored throughout the seizure, and arterial blood gases, $\mathrm{pH}$, and glucose were measured before the onset of seizures and at the start of brain freezing.

Ischemia experiment. Eighteen rats with unilateral LC lesion and 3 control rats were used in this experiment. Rats were decapitated by guillotine, and their heads were either immediately immersed and agitated in liquid nitrogen or incubated for periods of 1 and $2.5 \mathrm{~min}$ at $37^{\circ} \mathrm{C}$ before immersion in liquid nitrogen.

In a separate experiment, 4 control rats were decapitated and their heads were incubated at $37^{\circ} \mathrm{C}$ for $25 \mathrm{~min}$ before immersion in liquid nitrogen. This prolonged period of ischemia results in near total loss of brain tissue glycogen (Lowry et al., 1964). Samples from the cerebral cortex of these rats then were assayed for "glycogen" as described below to estimate the amount of glucose units liberated from non-glycogen sources (Lowry et al., 1964).

Preparation of cortical tissue and assay procedures. Rat heads were stored at $-70^{\circ} \mathrm{C}$ until they were sectioned coronally, $8 \mathrm{~mm}$ anterior to the lambda, using a Dremel band saw while liquid nitrogen was applied continuously to the saw blade and the frozen brain. The cerebral cortex from the posterior part was used for assays of metabolites and from the anterior part for assay of NE. Tissue cylinders were punched with an 18 gauge needle in a refrigerated glove box maintained at $-25^{\circ} \mathrm{C}$. Six to $8 \mathrm{mg}$ of cerebral cortical tissue was obtained in this fashion from bilaterally symmetrical medial and lateral locations as demonstrated in Figure 1. Care was taken to sample symmetrical areas restricted only to the cerebral cortex in all sections.

Tissue samples were weighed on a Cahn balance in the cold box and transferred to glass-glass homogenizer tubes containing $40 \mu \mathrm{l}$ of $0.1 \mathrm{~N} \mathrm{HCl}$ in $99 \%$ methanol kept at $-25^{\circ} \mathrm{C}$. Extraction of the tissue was performed according to the method of Folbergrova et al. (1972). The supernatant was neutralized and assayed for phosphocreatine, ATP, ADP, AMP, glucose, glucose 6-phosphate, fructose 1,6-diphosphate, pyruvate, and lactate by the methods of Lowry and Passonneau (1972) and for cyclic AMP by the

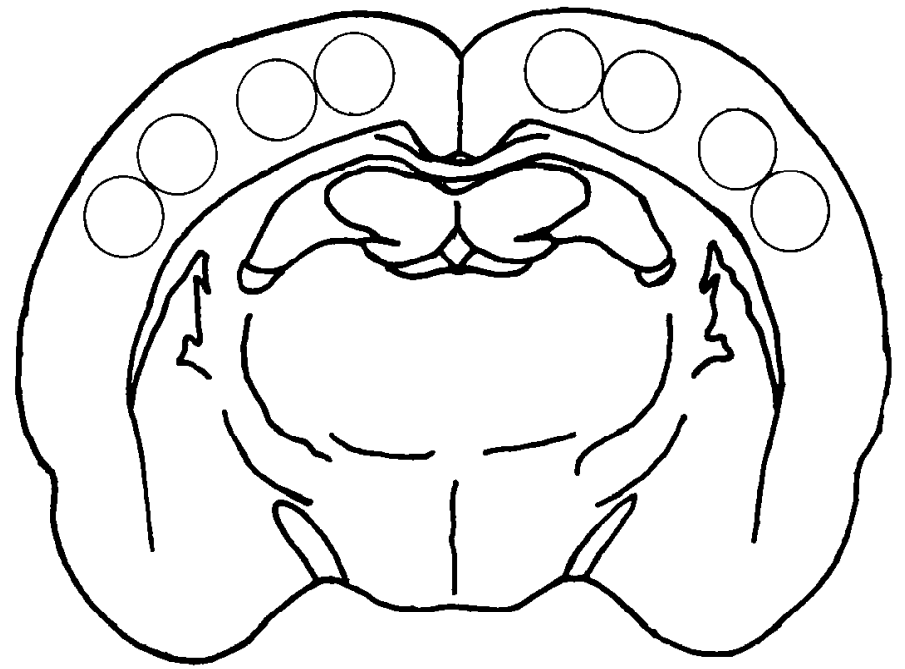

Figure 1. Schematic representation of the regions of the cerebral cortex (circles) that were punched out and used for assays of local cerebral metabolites. 
method of Gilman (1970). The precipitate was dispersed in $50 \mathrm{vol}$ (of the original weight of tissue) of $1 \mathrm{~N} \mathrm{HCl}$, the tube was sealed, and the glycogen content of the precipitate was hydrolyzed at $100^{\circ} \mathrm{C}$ for $2 \mathrm{hr}$. The hydrolysate then was neutralized and assayed for glucose (Lowry et al., 1964) as a reflection of the tissue glycogen content. Correction for the glucose liberated from non-glycogen sources was made by subtracting the amount of glucose obtained from samples of the cerebral cortex of rats that underwent 25 min of postdecapitation total cerebral ischemia. The amount of glucose liberated from these samples was $0.51 \pm 0.04 \mathrm{mmol} / \mathrm{kg}$ of tissue (mean $\pm \mathrm{SE}$ ). Cortical NE was assayed by the enzymatic-isotopic method of Henry et al. (1975).

Analysis of data. Data were analyzed by comparing the results obtained from the two hemispheres of each rat by the paired Student's $t$ test (two tailed) to minimize the effect of interanimal variations in seizure severity and differences in arterial blood gases, $\mathrm{pH}$, and glucose. Significance was considered at $p<0.05$.

\section{Results and Discussion}

For the purposes of this experiment, the "rest" conditions occurred $30 \mathrm{~min}$ after stopping halothane anesthesia, with the rat paralyzed and ventilated with $30 \%$ oxygen and $70 \%$ nitrous oxide and the surgical wounds infiltrated with local anesthetic solution. Cerebral cortical metabolites, NE levels, and values for arterial blood gases, $\mathrm{pH}$, and glucose that were obtained immediately before transcalvarial freezing are shown in Table I. Despite more than $90 \%$ depletion of $\mathrm{NE}$ in the ipsilateral cerebral cortex, there were no significant differences in cerebral metabolites between the contralateral and ipsilateral sides with the exception of glucose which was decreased by about $12 \%$ in the NE-depleted cortex $(p<$

TABLE I

Effects of unilateral locus ceruleus lesion on cerebral cortical metabolites under "rest" conditions

The values denote the means \pm SEM in millimoles per $\mathrm{kg}$ of tissue except for cyclic AMP and NE which are expressed as micromoles per $\mathrm{kg}$ and nanograms per $\mathrm{gm}$, respectively. $n$ is the number of observations obtained from 5 rats 2 weeks after unilateral LC lesion. Significance was considered at $p<0.05$ by the two-tailed paired Student's $t$ test. The mean arterial blood values \pm SEM, taken immediately before transcalvarial freezing, for the 5 rats in this experiment were: $\mathrm{PaCO}_{2}$, $38.3 \pm 0.8 \mathrm{~mm} \mathrm{Hg} ; \mathrm{PaO}_{2}, 141 \pm 8 \mathrm{~mm} \mathrm{Hg} ; \mathrm{pH} 7.48 \pm 0.02$; and glucose, $230 \pm 19 \mathrm{mg} \%$.

\begin{tabular}{lcccc}
\hline & $n$ & $\begin{array}{c}\text { Contralateral } \\
\text { Cortex }\end{array}$ & $\begin{array}{c}\text { Ipsilateral } \\
\text { Cortex }\end{array}$ & Significance \\
\hline Glycogen & 10 & $4.00 \pm 0.19$ & $4.09 \pm 0.21$ & NS $^{a}$ \\
$\begin{array}{l}\text { Glucose } \\
\text { Glucose 6-phos- }\end{array}$ & 10 & $2.51 \pm 0.22$ & $2.20 \pm 0.20$ & $p<0.05$ \\
$\quad$ phate & 10 & $0.09 \pm 0.01$ & $0.09 \pm 0.01$ & NS \\
Pyruvate & 10 & $0.20 \pm 0.02$ & $0.19 \pm 0.02$ & NS \\
Lactate & 9 & $0.97 \pm 0.07$ & $1.03 \pm 0.06$ & NS \\
Phosphocreatine & 8 & $4.19 \pm 0.31$ & $4.42 \pm 0.33$ & NS \\
ATP & 8 & $2.73 \pm 0.03$ & $2.73 \pm 0.08$ & NS \\
ADP & 8 & $0.36 \pm 0.02$ & $0.40 \pm 0.02$ & NS \\
AMP & 8 & $0.02 \pm 0$ & $0.02 \pm 0$ & NS \\
Cyclic AMP & 8 & $1.71 \pm 0.13$ & $1.68 \pm 0.19$ & NS \\
NE & 5 & $388 \pm 37$ & $33 \pm 15$ & $p<0.001$ \\
\hline
\end{tabular}

${ }^{a}$ NS, not significant.
0.05). The levels of metabolites shown in Table I are similar to our normal laboratory values for cortical tissue obtained from rats undergoing transcalvarial freezing under nitrous oxide anesthesia.

Seizures were provoked to study the effects of NE depletion under a condition of highly increased metabolic demand (Sacktor et al., 1966; Duffy et al., 1975; Chapman et al., 1977). EEG abnormalities first appeared about 30 $\mathrm{sec}$ after the intravenous injection of bicuculline. Isolated spikes were followed quickly by a tonic discharge lasting 20 to $40 \mathrm{sec}$. Subsequently, there were repeated bursts of spike and wave discharges separated by brief periods of electrical inactivity (Fig. 2). Seizure activity was similar on both sides in controls and in rats with unilateral LC lesions (Fig. 2). No attempts were made to control the rise in blood pressure which accompanied seizure activity. The magnitude of hypertension and the changes in arterial blood gases and glucose were similar in control and lesioned rats. Although rats were ventilated with $100 \%$ oxygen during seizures, $\mathrm{PaO}_{2}$ decreased to $79 \pm 6$ $\mathrm{mm} \mathrm{Hg}$ at the time of brain freezing. Other arterial blood findings showed $\mathrm{PaCO}_{2}$ increasing from $38.8 \pm 1.1$ to 44.2 $\pm 3.5 \mathrm{~mm} \mathrm{Hg}, \mathrm{pH}$ decreasing from $7.48 \pm 0.02$ to $7.31 \pm$ 0.04 , and plasma glucose increasing from $223 \pm 12$ to 270 $\pm 23 \mathrm{mg} \%$ during the seizure. In all rats, EEG recordings showed seizure activity until the termination of recording immediately prior to transcalvarial freezing.

The results of cortical metabolites and NE levels obtained from the seizure experiment are shown in Table II. There were no differences between the right and left sides of control rats, and thus, values from both sides of controls were lumped together. As reported previously (Sacktor et al., 1966; Duffy et al., 1975; Chapman et al., 1977), seizures cause definite changes in the cerebral metabolism of control rats: glycogen, glucose, and glucose 6-phosphate decreased by more than $50 \%$, while lactate increased about 6-fold. Phosphocreatine was decreased by about $50 \%$ and cyclic AMP was increased moderately, but ATP, ADP, and AMP levels were not changed (Tables I and II). Similar changes were induced by seizures in rats with unilateral LC lesion with two exceptions: in the NE-depleted cortex, glycogen decreased significantly less $(p<0.05)$ and cyclic AMP failed to increase (Table II).

Because of this apparent defect in glycogen breakdown and cyclic AMP accumulation in the NE-depleted cortex during seizures, we studied the effect of NE depletion on cerebral metabolites in total cerebral ischemia, a condition also associated with rapid glycogen breakdown (Lowry et al., 1964; Gatfield et al., 1966). It should be emphasized that, even when the rat head was immersed in liquid nitrogen immediately after decapitation, there is an estimated lag period of 30 to $35 \mathrm{sec}$ before the actual freezing of the cerebral cortex. Such estimation is based on the work of Swaab (1971) who used Freon 12 rather than liquid nitrogen in his freezing experiments. This explains the discrepancy in cerebral metabolite levels between rats that were frozen transcalvarially while their brains were being perfused as shown in Table I and those that were decapitated and their heads were immersed immediately in liquid nitrogen as shown in Table III. In the latter group, cerebral cortical levels of glucose were 

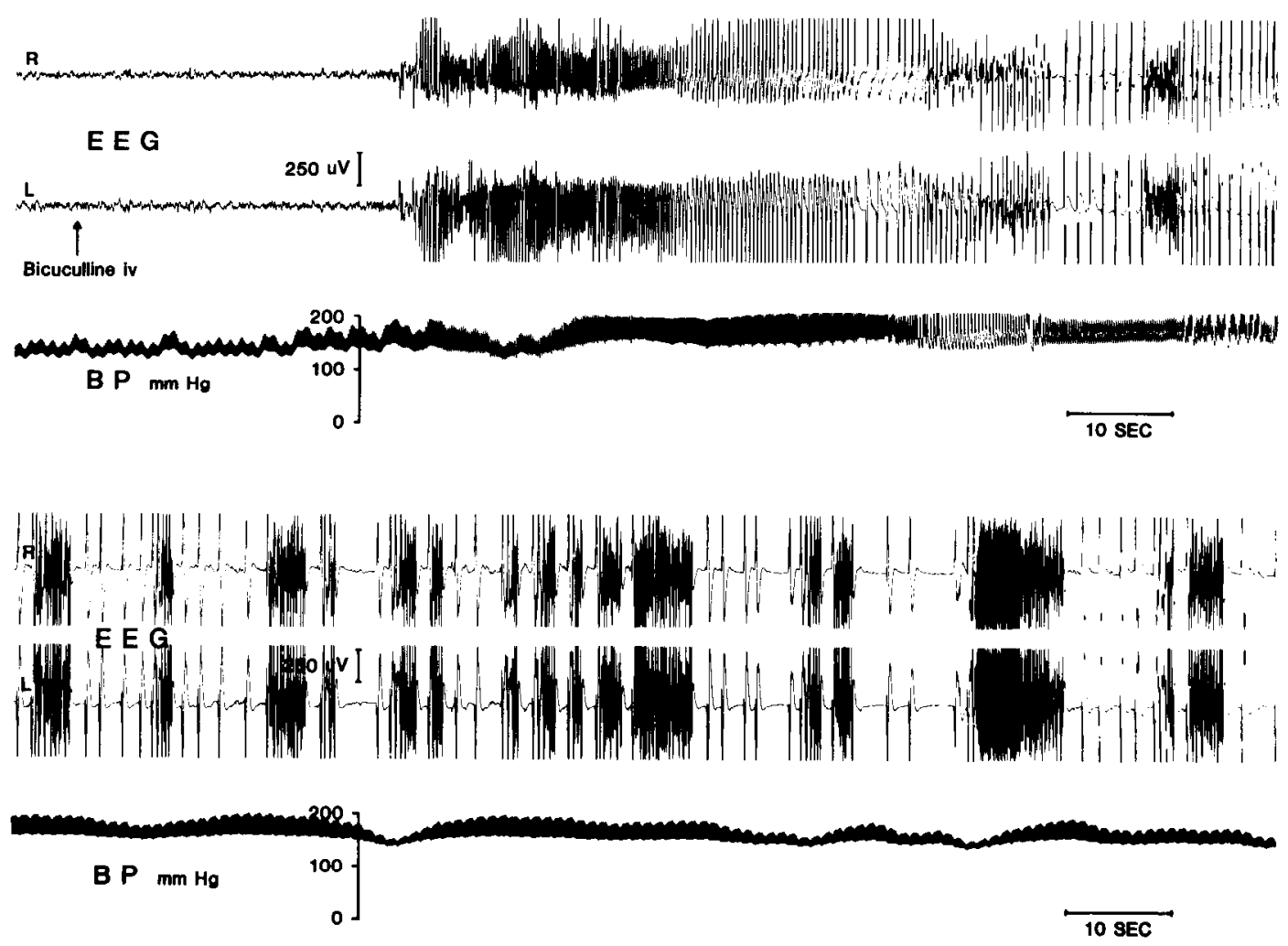

Figure 2. EEG and arterial blood pressure $(B P)$ recordings after the intravenous administration of bicuculline $(1.2 \mathrm{mg} / \mathrm{kg})$ to a rat 2 weeks after a right LC lesion. The rat was paralyzed and ventilated with $100 \%$ oxygen. The upper tracings describe the first $2 \mathrm{~min}$ and the lower tracings show the last 2 min of a 9-min seizure. NE levels in the right and left cerebral cortices were 16 and $260 \mathrm{ng} / \mathrm{gm}$ of tissue, respectively.

\section{TABLE II}

Effects of unilateral locus ceruleus lesion on cerebral cortical metabolites in bicuculline-induced seizures

The values denote the means \pm SEM in millimoles per $\mathrm{kg}$ of tissue except for cyclic AMP and NE which are expressed as micromoles per kg and nanograms per gm, respectively, $n$ is the number of observations obtained from 7 rats 2 weeks after unilateral LC lesion and 4 control rats. Significant differences between the contralateral and ipsilateral cortex were considered at $p<0.05$ by the two-tailed paired Student's $t$ test. Electrical seizures were maintained for 8 to $9 \mathrm{~min}$ before freezing. $\mathrm{PaO}_{2}$ decreased to $79 \pm 6 \mathrm{~mm} \mathrm{Hg}$ (mean of all 11 rats $\pm \mathrm{SEM}$ ) despite ventilation with $100 \% \mathrm{O}_{2}$ during seizures. $\mathrm{PaCO}_{2}$ increased from $38.8 \pm 1.1$ to $44.2 \pm 3.5 \mathrm{~mm} \mathrm{Hg}, \mathrm{pH}$ decreased from $7.48 \pm 0.2$ to $7.31 \pm 0.4$, and plasma glucose increased from $223 \pm 12$ to $270 \pm 23 \mathrm{mg} \%$ during seizures.

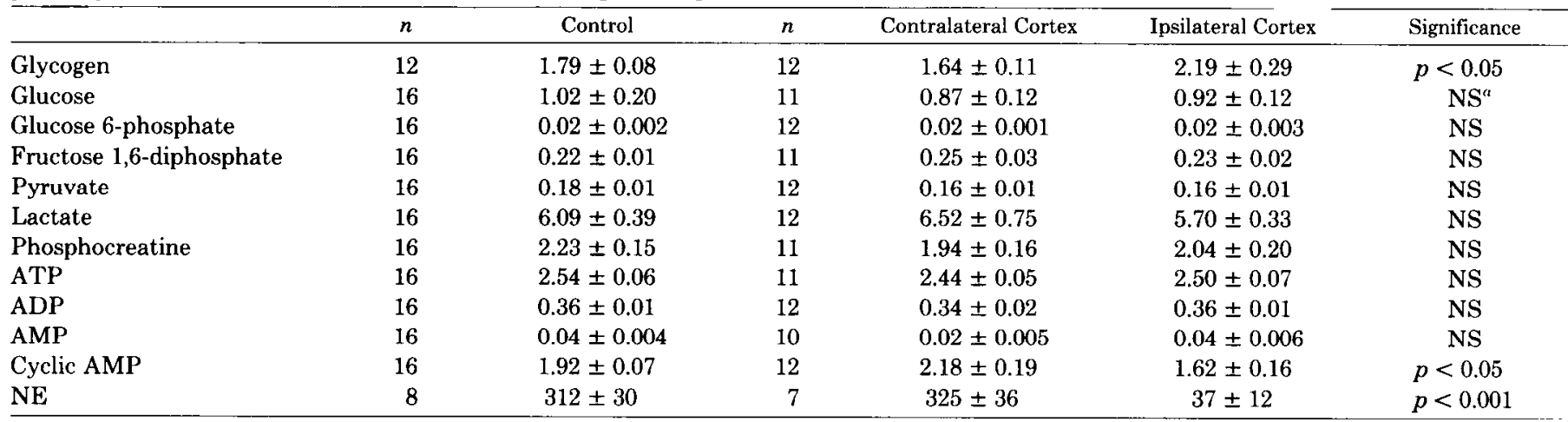

"NS, not significant.

depressed markedly, the lactate level increased about 4fold, and phosphocreatine declined to about $50 \%$ of the levels obtained when the brain was frozen transcalvarially. This emphasizes the fact that glucose, lactate, and phosphocreatine are the most sensitive indicators of cerebral ischemia.

In the ischemia experiment, the results from the cortex contralateral to LC lesion and those from control rats (results not shown) are similar to previous results of postdecapitation ischemia studies (Lowry et al., 1964; Gatfield et al., 1966). Despite about 90 to $95 \%$ depletion of $\mathrm{NE}$ in the ipsilateral cortex, there were no significant differences between the ipsilateral and contralateral cortex when rat heads were immersed in liquid nitrogen 
TABLE III

Effects of unilateral locus ceruleus lesion on cerebral cortical metabolites during ischemia

Two weeks after LC lesion, 5 to 8 rats in each group were decapitated by guillotine and their heads were either $(1)$ immediately immersed in liquid nitrogen, (2) incubated for $1 \mathrm{~min}$, or (3) incubated for $2.5 \mathrm{~min}$ at $37^{\circ} \mathrm{C}$ prior to immersion in liquid nitrogen. The values denote the means of 9 to 16 observations for metabolites and 5 to 8 observations for NE \pm SEM in millimoles per kg of tissue except for cyclic AMP and NE which are expressed in micromoles per $\mathrm{kg}$ and nanograms per gm, respectively. Significant differences between the ipsilateral and contralateral cortex were assessed by the paired Student's $t$ test (two tailed) (see footnotes).

\begin{tabular}{|c|c|c|c|c|c|c|}
\hline & \multicolumn{6}{|c|}{ Time Interval between Decapitation and Immersion of Head in Liquid Nitrogen } \\
\hline & \multicolumn{2}{|c|}{ Immediately } & \multicolumn{2}{|c|}{$1 \mathrm{Min}$} & \multicolumn{2}{|c|}{$2.5 \mathrm{Min}$} \\
\hline & $\begin{array}{c}\text { Ipsilateral } \\
\text { Cortex }\end{array}$ & $\begin{array}{c}\text { Contralateral } \\
\text { Cortex }\end{array}$ & $\begin{array}{c}\text { Ipsilateral } \\
\text { Cortex }\end{array}$ & $\begin{array}{c}\text { Contralateral } \\
\text { Cortex }\end{array}$ & $\begin{array}{c}\text { Ipsilateral } \\
\text { Cortex }\end{array}$ & $\begin{array}{c}\text { Contralateral } \\
\text { Cortex }\end{array}$ \\
\hline Glycogen & $3.91 \pm 0.30$ & $3.46 \pm 0.17$ & $2.03 \pm 0.16^{a}$ & $1.66 \pm 0.11$ & $1.31 \pm 0.13^{b}$ & $1.05 \pm 0.12$ \\
\hline Glucose & $0.16 \pm 0.03$ & $0.16 \pm 0.04$ & $0.13 \pm 0.02$ & $0.15 \pm 0.03$ & $0.04 \pm 0.01$ & $0.06 \pm 0.02$ \\
\hline Pyruvate & $0.17 \pm 0.02$ & $0.14 \pm 0.01$ & $0.06 \pm 0.00$ & $0.05 \pm 0.00$ & $0.08 \pm 0.01$ & $0.08 \pm 0.01$ \\
\hline Lactate & $1.09 \pm 0.26$ & $3.72 \pm 0.26$ & $7.19 \pm 0.39$ & $7.21 \pm 0.13$ & $9.79 \pm 0.23$ & $9.62 \pm 0.35$ \\
\hline Phosphocreatine & $2.11 \pm 0.13$ & $1.91 \pm 0.09$ & $0.23 \pm 0.04$ & $0.29 \pm 0.04$ & & \\
\hline ATP & $2.59 \pm 0.11$ & $2.50 \pm 0.19$ & $0.27 \pm 0.03$ & $0.28 \pm 0.04$ & $0.25 \pm 0.05$ & $0.21 \pm 0.04$ \\
\hline ADP & $0.42 \pm 0.11$ & $0.44 \pm 0.07$ & $1.70 \pm 0.08$ & $1.78 \pm 0.05$ & $0.55 \pm 0.03$ & $0.60 \pm 0.03$ \\
\hline AMP & $0.05 \pm 0.01$ & $0.05 \pm 0.01$ & $0.69 \pm 0.02$ & $0.67 \pm 0.01$ & $0.72 \pm 0.01$ & $0.72 \pm 0.01$ \\
\hline Cyclic AMP & $2.39 \pm 0.16$ & $2.78 \pm 0.14$ & $2.59 \pm 0.11^{a}$ & $3.40 \pm 0.22$ & $2.10 \pm 0.14^{a}$ & $2.92 \pm 0.27$ \\
\hline $\mathrm{NE}$ & $37 \pm 8^{c}$ & $327 \pm 30$ & $22 \pm 3^{c}$ & $344 \pm 20$ & $19 \pm 3^{c}$ & $340 \pm 28$ \\
\hline
\end{tabular}

" $p<0.01$.

${ }^{b} p<0.05$.

${ }^{c} p<0.001$.

immediately after decapitation. Although glycogen levels were elevated by about $15 \%$ in the ipsilateral cortex, in comparison to the contralateral cortex, the difference between the two hemispheres did not reach statistical significance $(p>0.05)$. However, when the period of ischemia was prolonged by 1 and $2.5 \mathrm{~min}$, then there was a statistically significant difference between the two sides in the levels of glycogen and cyclic AMP, with the former being higher by about $25 \%$ and the latter being lower in the NE-depleted cortex (Table III). No other differences in cerebral metabolites were seen between the ipsilateral and contralateral sides.

Our results indicate that depletion of NE in the cerebral cortex has little effect on cerebral metabolites under "rest" conditions. The only exception is the modest, but significant, reduction in glucose levels in the NE-depleted cortex. The cause of this finding is not apparent at the present time. These results are in agreement with Hoffmann et al. (1973) and Korf and Sebens (1979) who reported that basal levels of cerebral glycogen and cyclic AMP are not altered by NE depletion.

The most important finding of this study is that glycogen levels remain significantly higher in the NE-depleted cortex under two conditions known to be associated with rapid glycogenolysis. Higher glycogen levels ipsilateral to LC lesion can result from the decreased energy demand by the NE-depleted cortex during seizures and ischemia or from the decreased ability of the NE-depleted cortex to utilize glycogen despite high demand. At least in seizures, the first possibility is unlikely, since cortical NE depletion, whether by drugs (Gross and Ferrendelli, 1979) or by lesion of ascending noradrenergic pathways (Mason and Corcoran, 1979), enhances seizure activity. Furthermore, electrical seizure activity was bilaterally symmetrical in our rats with unilateral LC lesion (Fig. 2). The more likely explanation is that cerebral glycogen breakdown is hindered by NE depletion during both seizures and ischemia. This explanation agrees well with our previous findings of delayed re-reduction of NAD and cytochrome $a, a_{3}$ after these mitochondrial components were oxidized transiently in response to brief electrical stimulation of the cerebral cortex (Harik et al., 1979; LaManna et al., 1981). Increased lactate accumulation in the contralateral cerebral cortex during ischemia would have added strength to our explanation, but contrary to our expectations, there were no significant differences in lactate between the two sides. The associated lack of increased cyclic AMP levels after seizures and the modest increase during ischemia in the NE-depleted cortex is also compatible with previous reports (Gross and Ferrendelli, 1979; Folbergrova, 1977) and suggests that cyclic AMP may modulate glycogenolysis by activating glycogen phosphorylase.

\section{References}

Chapman, A. G., B. S. Meldrum, and B. K. Siesjö (1977) Cerebral metabolic changes during prolonged epileptic seizures in rats. J. Neurochem. 28: 1025-1035.

Duffy, T. E., D. C. Howse, and F. Plum (1975) Cerebral energy metabolism during experimental status epilepticus. J. Neurochem. 24: 925-933.

Edwards, C., S. R. Nahorski, and K. J. Rogers (1974) In vivo changes of cerebral cyclic AMP induced by biogenic amines: Association with phosphorylase activation. J. Neurochem. 22: 565-572.

Estler, C. J., and H. P. T. Ammon (1967) The influence of propranolol on the methamphetamine-induced changes of cerebral function and metabolism. J. Neurochem. 14: 799805.

Folbergrova, J. (1977) Changes of cyclic AMP and phosphorylase $a$ in mouse cerebral cortex during seizures induced by 3-mercaptopropionic acid. Brain Res. 135: 337-346.

Folbergrova, J., V. MacMillan, and B. K. Siesjö (1972) The effect of hypercapnic acidosis upon some glycolytic and Kreb's cycle-associated intermediates in the rat brain. J. Neurochem. 19: 2507-2517. 
Gatfield, P. D., O. H. Lowry, D. W. Schulk, and J. V.Passonneau (1966) Regional energy reserves in mouse brain and changes with ischemia and anesthesia. J. Neurochem. 13: 185-195.

Gilman, A. G. (1970) A protein binding assay for adenosine 3':5'-cyclic monophosphate. Proc. Natl. Acad. Sci. U. S. A. 67: $305-312$.

Gross, R. A., and J. A. Ferrendelli (1979) Effects of reserpine, propranolol, and aminophylline on seizure activity and CNS cyclic nucleotides. Ann. Neurol. 6: 296-301.

Gross, R. A., and J. A. Ferrendelli (1980) Mechanisms of cyclic AMP regulation in cerebral anoxia and their relationship to glycogenolysis. J. Neurochem. 34: 1309-1318.

Harik, S. I., J. C. LaManna, A. I. Light, and M. Rosenthal (1979) Cerebral norepinephrine: Influence on cortical oxidative metabolism in situ. Science 206: 69-71.

Harik, S. I., R. B. Duckrow, J. C. LaManna, M. Rosenthal, V. K. Sharma, and S. P. Banerjee (1981) Cerebral compensation for chronic noradrenergic denervation induced by locus ceruleus lesion: Recovery of receptor binding, isoproterenol-induced adenylate cyclase activity, and oxidative metabolism. J. Neurosci. 1: 641-649.

Henry, D. P., B. J. Starman, D. G. Johnson, and R. H. Williams (1975) A sensitive radioenzymatic assay for norepinephrine in tissue and plasma. Life Sci. 16: 375-384.

Hoffmann, P. C., R. Toon, J. Kleinman, and A. Heller (1973) The association of lesion-induced reductions in brain monoamines with alterations in striatal carbohydrate metabolism. J. Neurochem. 20: 69-80.

Kobayashi, R. M., M. Palkovits, D. M. Jacobowitz, and I. J. Kopin (1975) Biochemical mapping of the noradrenergic pro- jection from the locus ceruleus. Neurology (N. Y.) 25: 223233.

Korf, J., and J. B. Sebens (1979) Cyclic AMP in the rat cerebral cortex after activation of noradrenaline neurons of the locus coeruleus. J. Neurochem. 32: 463-468.

LaManna, J. C., S. I. Harik, A. I. Light, and M. Rosenthal (1981) Norepinephrine depletion alters cerebral oxidative metabolism in the "active" state. Brain Res. 204: 87-101.

Lowry, O. H., and J. V. Passonneau (1972) A Flexible System of Enzymatic Analysis, Academic Press, New York.

Lowry, O. H., J. V. Passonneau, F. X. Hasselberger, and D. W. Schulz (1964) Effect of ischemia on known substrates and cofactors of the glycolytic pathway in brain. J. Biol. Chem. 239: $18-30$.

Mason, S. T., and M. E. Corcoran (1979) Catecholamines and convulsions. Brain Res. 170: 497-507.

Passonneau, J. V., E. A. Brunner, C. Molstad, and R. Passoneau (1971) The effects of altered endocrine states and of ether anaesthesia on mouse brain. J. Neurochem. 18: 2317-2328.

Ponten, U., R. A. Ratcheson, L. G. Salford, and B. K. Siesjö (1973) Optimal freezing conditions for cerebral metabolites in rats. J. Neurochem. 21: 1127-1138.

Sacktor, B., J. E. Wilson, and C. G. Teikert (1966) Regulation of glycolysis in brain, in situ, during convulsions. J. Biol. Chem. 241: 5071-5075.

Swaab, D. F. (1971) Pitfalls in the use of rapid freezing for stopping brain and spinal cord metabolism in rat and mouse. J. Neurochem. 18: 2085-2092.

Ungerstedt, U. (1971) Stereotaxic mapping of monoamine pathways in the rat brain. Acta Physiol. Scand. Suppl. 367: 1-48. 